\title{
AN INCREMENTAL APPROACH FOR THE IDENTIFICATION OF REACTION KINETICS
}

\author{
Marc Brendel*, Adel Mhamdi*, \\ Dominique Bonvin ${ }^{* *}$, Wolfgang Marquardt ${ }^{*, 1}$ \\ * Lehrstuhl für Prozesstechnik, RWTH Aachen University, \\ D-52064 Aachen, Germany \\ ** Laboratoire d'Automatique, EPFL, CH-1015 Lausanne, \\ Switzerland
}

\begin{abstract}
This paper proposes an incremental approach for the identification of complex reaction kinetics in chemical reactors. The reaction fluxes for the various species are first estimated on the basis of concentration measurements and balance equations. This task represents an ill-posed inverse problem requiring appropriate regularization. In a further step, the reaction rates are estimated without postulating a kinetic structure. Finally, the dependency of the reaction rates on concentrations, i.e. the kinetic laws, are constructed by means of feedforward neural networks. This incremental approach is shown to be both efficient and flexible for utilizing the available process knowledge. The methodology is illustrated on the industrially-relevant acetoacetylation of pyrrole with diketene.
\end{abstract}

Keywords: Identification, reactor modeling, input estimation, regularization, neural networks

\section{INTRODUCTION}

The description of reaction kinetics often represents the most challenging part in the modeling of chemical reactors. A reliable description is rarely available a priori. For example, it is well known that reaction kinetics cannot necessarily be derived from stoichiometries (Connors, 1990), in particular in the case of catalyzed reactions. Thus, a reliable kinetic model needs to be identified from experimental data.

The model-based techniques used in process control and optimization require a model that adequately describes the process dynamics, i.e. also the kinetics in reaction systems. For the case where a kinetic structure is not available, Psichogios and Ungar (1992) proposed a hybrid ap-

1 Corresponding author. E-mail: marquardt@lfpt.rwthaachen.de proach to process modeling as an alternative to recurrent neural networks for describing the dynamic system. The hybrid model combines prior knowledge on mass and energy balances with a feed-forward neural net model that serves as a substitute for the constitutive equations that cannot be determined from first principles. These authors found that the hybrid model has better properties than standard black-box neural net models, i.e. interpolation and extrapolation are more accurate and the model is easier to analyze and interpret. Parameters in the neural net part of the hybrid model can be estimated from experimental data. Recently, Tholudur and Ramirez (1999) used a two-step approach for the identification of kinetics: Reaction rates are first identified, assuming known curve characteristics, and subsequently correlated with the independent state variables using a feed-forward neural net approximation. Van Lith et al. (2002) combined 
an extended Kalman filter for the estimation of states and rates with subsequent fuzzy submodel identification.

In this work, an incremental approach for the identification of reaction kinetics is proposed when no prior kinetic knowledge is available. The approach is applicable to all reactor types, i.e. also to those exhibiting transient behavior and possibly variable feed and effluent streams. The reaction fluxes for the various species are estimated from noisy concentration data using the approach of Mhamdi and Marquardt (1999). Then, the individual reaction rates can be calculated using knowledge of reaction stoichiometry. These reaction rates and the concentration data serve as input to a Bayesian algorithm to train a feedforward neural network yielding the kinetic model. The approach is especially suited for nowaday's high resolution measurement techniques such as IR (Alsmeyer et al., 2002) or Raman spectroscopy (Bardow et al., 2003), where concentration data can be obtained continuously in-situ.

\section{PRELIMINARIES}

\subsection{Model of the reaction system}

Consider a homogeneous, not necessarily isothermal, chemical reaction system with $R$ reactions involving $S$ species. The time evolution of the number of moles of species $i, n_{i}[\mathrm{~mol}]$, is given by:

$$
\frac{\mathrm{d} n_{i}}{\mathrm{~d} t}=f_{i}^{\text {in }}-f_{i}^{\text {out }}+f_{i}^{\mathrm{r}}, \quad i=1, . ., S
$$

where $f_{i}^{\text {in }}$ and $f_{i}^{\text {out }}[\mathrm{mol} / \mathrm{min}]$ are the molar flow rates of species $i$ into and out of the reactor and $f_{i}^{\mathrm{r}}[\mathrm{mol} / \mathrm{min}]$ is the reaction flux of species $i$, i.e. the net molar flow rate of species $i$ produced or consumed by the various chemical reactions.

The reaction flux of species $i$ can be expressed in terms of the individual reaction rates:

$$
f_{i}^{\mathrm{r}}=V \sum_{j}^{R} \nu_{i j} r_{j}, \quad i=1, . ., S
$$

where $\nu_{i j}$ is the stoichiometric coefficient for species $i$ in the $j$ th reaction, $r_{j}[\mathrm{~mol} / \mathrm{l} \mathrm{min}]$ the rate of the $j$ th reaction, and $V$ [l] the volume.

In vector form, equation (2) reads:

$$
\mathbf{f}^{\mathrm{r}}=V \mathbf{N r}
$$

where $\mathbf{f}^{\mathrm{r}}$ is the $S$-dimensional reaction flux vector, $\mathbf{r}$ the $R$-dimensional reaction rate vector and $\mathbf{N}$ the $S \times R$ stoichiometric matrix.

Equation (3) indicates that, if $S \geq R$, the reaction rate vector can be calculated from the reaction fluxes as follows:

$$
\mathbf{r}=\frac{1}{V} \mathbf{N}^{+} \mathbf{f}^{\mathrm{r}}
$$

where $\mathbf{N}^{+}$is the Moore-Penrose inverse of $\mathbf{N}$.

For a constant-density semi-batch reactor with a volumetric feed of rate $F[\mathrm{l} / \mathrm{min}]$ and concentration $c_{i}^{\text {in }}[\mathrm{mol} / \mathrm{l}]$ and no outflow, the mole balance equation (1) expressed in terms of the molar concentration $c_{i}=n_{i} / V[\mathrm{~mol} / \mathrm{l}]$, and the total mass balance give

$$
\begin{aligned}
\frac{\mathrm{d} c_{i}}{\mathrm{~d} t} & =\frac{F}{V}\left(c_{i}^{\mathrm{in}}-c_{i}\right)+\frac{f_{i}^{\mathrm{r}}}{V} \\
\frac{\mathrm{d} V}{\mathrm{~d} t} & =F,
\end{aligned}
$$

implying no volume change by the reactions.

\subsection{Estimation of reaction fluxes}

The reaction fluxes $f_{i}^{\mathrm{r}}(t)$ can be estimated independently for each species. A generic model of the problem is developed as follows. Let

$$
y_{i}(t)=n_{i}(t)-n_{i}\left(t_{0}\right)-\int_{t_{0}}^{t} u_{i}(\tau) d \tau,
$$

where $u_{i}(\tau)=f_{i}^{\text {in }}(\tau)-f_{i}^{\text {out }}(\tau)$. This transformation, applied to $(1)$, leads to

$$
\frac{\mathrm{d} y_{i}(t)}{\mathrm{d} t}=f_{i}^{\mathrm{r}}(t), y_{i}\left(t_{0}\right)=0
$$

where $f_{i}^{\mathrm{r}}(t)$ is considered as an unknown input that must be determined on the basis of a noisy measurement

$$
\tilde{y}_{i}(t)=y_{i}(t)+\varepsilon_{y_{i}}(t) .
$$

Here, the superscript $(\tilde{*})$ is used to denote a noisy quantity and $\varepsilon_{y}$ represents the measurement noise contained in $\tilde{y}$.

This estimation problem represents an ill-posed inverse problem according to the definition of Hadamard (Engl et al., 1996). Since the measurement is noisy, the estimate $\hat{f}_{i}^{\mathrm{r}}(t)$ of $f_{i}^{\mathrm{r}}(t)$ can be arbitrarily large if no regularization of the solution is considered. Mhamdi and Marquardt (1999) used Tikhonov-Arsenin filtering for the estimation of $f_{i}^{\mathrm{r}}(t)$. The quality of the estimation is greatly influenced by the choice of the regularization parameter that weighs the tradeoff between noise reduction and bias in the estimate. Adequate regularization parameters can be determined by the L-curve criterion (Hansen, 1998), for example.

Another approach to filtering is the use of smoothing splines (Craven and Wahba, 1979). Splines are piecewise polynomial functions that possess certain smoothness and differentiability properties at the nodes. General cross validation $(G C V)$ is often used to select a suitable regularization parameter (Craven and Wahba, 1979). 


\section{INCREMENTAL IDENTIFICATION APPROACH}

The incremental identification approach mirrors the steps taken when developing a model for a given process. During model development, the balance equations are set up first and the unknown fluxes are then described by constitutive equations. If needed, variable parameters in the constitutive equations can be modeled as functions of the system states. Transferring this procedure to the identification process, the incremental identification approach features the stepwise identification of quantities as they are used in the modeling process. In an adaptive model identification context (Marquardt, 2002), the incremental approach allows the utilization of as much information as can be safely provided by first-principle modeling or sound empirical approaches. The process of identification then reduces to modeling uncertainties, i.e. unknown parameters in a given structure or the model structure itself. This way, the identification procedure is split up into a sequence of decoupled identification problems. This offers two main advantages: i) the solution at a given step becomes more simple as e.g. process dynamics are considered in the first step and can be omitted subsequently, and ii) physical insight is provided for tackling the following steps.

The incremental identification approach for the identification of reaction kinetics is depicted in Figure 1. It includes the following steps:

(1) The fluxes $\hat{f}_{i}^{\mathrm{r}}(t), \mathrm{i}=1, . ., S$ are estimated using mole balances (Model 1). Use equations (7)(9) and $\tilde{n}_{i}=\tilde{c}_{i} V$.

(2) With additional information on stoichiometry (Model 2), the reaction rates $\hat{r}_{j}(t)$, $\mathrm{j}=1, . ., R$ are then calculated using (4).

(3) Furthermore, if the rate laws (e.g. $r=$ $k c_{A} c_{B}$ ) are known (Model 3), (time-variant) rate constants $\hat{k}_{j}(t)$ are calculated from the reaction rate $\hat{r}_{j}(t)$ and concentrations $\hat{c}_{i}(t)$.

(4) Model 4 in addition assumes a temperature dependency of $k$ such as the Arrhenius law $\left(k=k_{0} e^{\frac{-E}{R T}}\right)$. The rate constant parameters $\left(\hat{k}_{0 j}, \hat{E}_{j}\right)$ can then be estimated from $\hat{k}_{j}(t)$ and $\hat{T}(t)$.

If parts of the kinetics are unknown, such as the Arrhenius law, the outputs of Model 3 can be taken as inputs to a data-driven approach for describing $k=k(T)$. For an unknown rate law (Model 2 known), $\hat{c}_{i}, T$ and $\hat{r}_{j}$ serve as inputs to the data-driven models $r_{j}=r_{j}\left(c_{i}\right)$ and $r_{j}=$ $r_{j}\left(c_{i}, T\right)$ for the isothermal and non-isothermal cases, respectively. If the reaction stoichiometry is unknown, target factor analysis (Bonvin and Rippin, 1990) can help identify the stoichiometry based on the estimated fluxes.

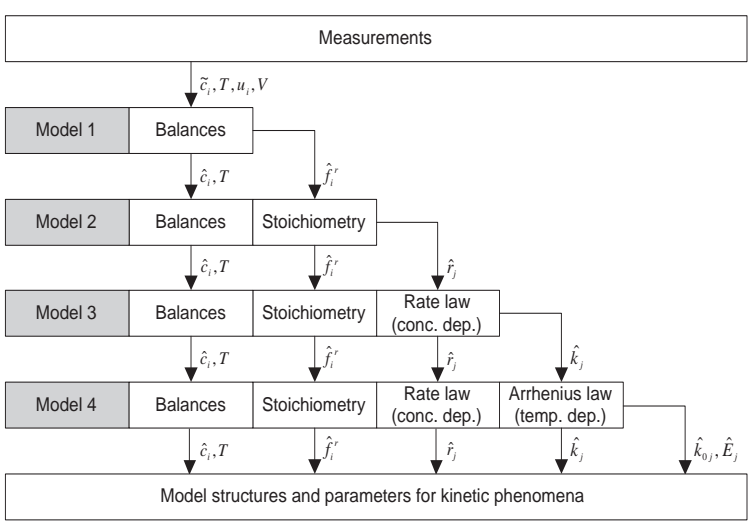

Fig. 1. Incremental approach for reaction kinetics identification

For describing functional relations in a data set, methods can be grouped in two categories based on the quality and amount of prior knowledge. If a model structure is available, the unknown model parameters can be identified from the data. Lacking such a model structure, black-box approaches are usually used, with the choice of basis functions based on some prior knowledge. Their ability to approximate any function arbitrarily well, given a sufficient number of parameters, may imply overfitting where the error on the training set is small, but a large error results if new data are presented to the model. To avoid overfitting and improve the predictive capability of the model, regularization, model discrimination or data validation techniques are commonly used. Feed-forward neural networks with Bayesian regularization (MacKay, 1992) may serve as an automated regularization procedure for training.

\section{ILLUSTRATIVE EXAMPLE}

\subsection{Simulated reaction system}

The incremental approach for identifying reaction kinetics is illustrated on the acetoacetylation of pyrrole with diketene (Ruppen et al., 1997):

$$
\begin{aligned}
& \mathrm{P}+\mathrm{D} \stackrel{\mathrm{K}}{\rightarrow} \mathrm{PAA} \\
& \mathrm{D}+\mathrm{D} \stackrel{\mathrm{K}}{\rightarrow} \mathrm{DHA} \\
& \mathrm{D} \rightarrow \text { oligomers } \\
& \mathrm{PAA}+\mathrm{D} \stackrel{\mathrm{K}}{\rightarrow} \mathrm{F}
\end{aligned}
$$

In addition to the desired reaction of diketene (D) and pyrrole $(\mathrm{P})$ to 2-acetoacetyl pyrrole (PAA) (10a), there are several undesired side reactions (10b)-(10d). These include the dimerization and oligomerization of diketene to dehydroacetic acid (DHA) and oligomers as well as a consecutive reaction to the by-product $\mathrm{F}$. The reactions take place isothermally in a laboratory-scale semibatch reactor with an initial volume of 1 liter, to which a diluted solution of diketene is added continuously. 
Reactions (10a), (10b) and (10d) are catalyzed by pyridine $(\mathrm{K})$, the concentration of which continuously decreases during the run due to addition of the diluted diketene feed. The dilution of catalyst is modeled by normalizing the corresponding rate constants with respect to the volume. Reaction (10c), which is assumed to be promoted by other intermediate products, is not normalized. Hence, the effective reaction rates are described by the following constitutive equations

$$
\begin{aligned}
r_{j}(t) & =\frac{V_{0}}{V(t)} r_{j}^{\star}(t), \quad j=\{a, b, d\}, \\
r_{c}(t) & =r_{c}^{\star}(t),
\end{aligned}
$$

with the formal reaction rates

$$
\begin{aligned}
& r_{a}^{\star}(t)=k_{a} c_{\mathrm{P}}(t) c_{\mathrm{D}}(t), \\
& r_{b}^{\star}(t)=k_{b} c_{\mathrm{D}}^{2}(t), \\
& r_{c}^{\star}(t)=k_{c} c_{\mathrm{D}}(t), \\
& r_{d}^{\star}(t)=k_{d} c_{\mathrm{PAA}}(t) c_{\mathrm{D}}(t),
\end{aligned}
$$

where $k_{a}, k_{b}, k_{c}$ and $k_{d}$ represent the rate constants and $V_{0}$ the initial volume.

The mole balances for the species D, P, PAA and DHA read

$$
\begin{aligned}
& \frac{\mathrm{d} c_{\mathrm{D}}(t)}{\mathrm{d} t}=\frac{F(t)}{V(t)}\left[c_{\mathrm{D}}^{\mathrm{in}}-c_{\mathrm{D}}(t)\right]+\frac{f_{\mathrm{D}}^{\mathrm{r}}(t)}{V(t)}, \\
& \frac{\mathrm{d} c_{\mathrm{P}}(t)}{\mathrm{d} t}=-\frac{F(t)}{V(t)} c_{\mathrm{P}}(t)+\frac{f_{\mathrm{P}}^{\mathrm{r}}(t)}{V(t)} \\
& \frac{\mathrm{d} c_{\mathrm{PAA}}(t)}{\mathrm{d} t}=-\frac{F(t)}{V(t)} c_{\mathrm{PAA}}(t)+\frac{f_{\mathrm{PAA}}^{\mathrm{r}}(t)}{V(t)}, \\
& \frac{\mathrm{d} c_{\mathrm{DHA}}(t)}{\mathrm{d} t}=-\frac{F(t)}{V(t)} c_{\mathrm{DHA}}(t)+\frac{f_{\mathrm{DHA}}^{\mathrm{r}}(t)}{V(t)},
\end{aligned}
$$

with the initial conditions $c_{\mathrm{D}}(0)=c_{\mathrm{D} 0}, c_{\mathrm{P}}(0)=$ $c_{\mathrm{P} 0}, c_{\mathrm{PAA}}(0)=c_{\mathrm{PAA} 0}$ and $c_{\mathrm{DHA}}(0)=c_{\mathrm{DHA} 0}$. The reaction fluxes $f_{\mathrm{D}}^{\mathrm{r}}, f_{\mathrm{P}}^{\mathrm{r}}, f_{\mathrm{PAA}}^{\mathrm{r}}$ and $f_{\mathrm{DHA}}^{\mathrm{r}}$ can be related to the reaction rates using the stoichiometry:

$$
\begin{aligned}
& f_{\mathrm{D}}^{\mathrm{r}}=\left(-r_{a}-2 r_{b}-r_{c}-r_{d}\right) V, \\
& f_{\mathrm{P}}^{\mathrm{r}}=-r_{a} V, \\
& f_{\mathrm{PAA}}^{\mathrm{r}}=\left(r_{a}-r_{d}\right) V, \\
& f_{\mathrm{DHA}}^{\mathrm{r}}=r_{b} V .
\end{aligned}
$$

\subsection{Experimental design}

To assess the capability of the incremental identification approach and allow a comparison of the modeled and true kinetics, concentration trajectories are generated using the model described above and the rate constants given in Table 1.

The measured concentrations are assumed to stem from a high-resolution in-situ measurement technique such as Raman spectroscopy, taken at a
Table 1 . Values of rate constants

\begin{tabular}{ccccc}
\hline & $\begin{array}{c}k_{a} \\
{\left[\frac{l}{\text { molmin }}\right]}\end{array}$ & $\begin{array}{c}k_{b} \\
{\left[\frac{l}{\text { molmin }}\right]}\end{array}$ & $\begin{array}{c}k_{c} \\
{\left[\frac{1}{\min }\right]}\end{array}$ & $\begin{array}{c}k_{d} \\
{\left[\frac{l}{\operatorname{molmin}}\right]}\end{array}$ \\
\hline value & 0.053 & 0.128 & 0.028 & 0.001 \\
\hline
\end{tabular}

Table 2. Range of independent variables

\begin{tabular}{ccccccc}
\hline & $\begin{array}{c}c_{\mathrm{D} 0} \\
{\left[\frac{m o l}{l}\right]}\end{array}$ & $\begin{array}{c}c_{\mathrm{P0}} \\
{\left[\frac{m o l}{l}\right]}\end{array}$ & $\begin{array}{c}c_{\mathrm{PAA0}} \\
{\left[\frac{m o l}{l}\right]}\end{array}$ & $\begin{array}{c}c_{\mathrm{DHA0}} \\
{\left[\frac{\mathrm{mol}}{l}\right]}\end{array}$ & $\begin{array}{c}F \\
{\left[\frac{l}{m i n}\right]}\end{array}$ & $\begin{array}{c}c_{\mathrm{D}}^{\text {in }} \\
{\left[\frac{m o l}{l}\right]}\end{array}$ \\
\hline $\min$ & 0.07 & 0.40 & 0.10 & 0.02 & $0.5 \mathrm{e}-3$ & 4.0 \\
$\max$ & 0.14 & 0.80 & 0.20 & 0.04 & $1.5 \mathrm{e}-3$ & 6.0 \\
\hline
\end{tabular}

sampling frequency $f_{\mathrm{s}}=60 \mathrm{~min}^{-1}$ and corrupted with normally distributed white noise of standard deviation $\sigma_{\mathrm{c}}=0.01 \mathrm{~mol} / \mathrm{l}$. The batch time is $t_{\mathrm{f}}=60 \mathrm{~min}$. Concentration data are available for the species D, P, PAA and DHA, but not for the oligomers and the side product $\mathrm{F}$ since the latter are difficult to obtain.

The reaction system (10a)-(10d) suggests that $r_{b}$ and $r_{c}$ are univariate functions of $c_{\mathrm{D}}$, whereas $r_{a}$ and $r_{d}$ are expected to be bivariate functions of $c_{\mathrm{P}}, c_{\mathrm{D}}$ and $c_{\mathrm{PAA}}, c_{\mathrm{D}}$, respectively.

To obtain reliable approximations of the reaction rates, in particular for the bivariate functions, experiments are designed so as to obtain concentration data over a large domain. Six independent variables can be considered: the four initial conditions $c_{\mathrm{D} 0}, c_{\mathrm{P} 0}, c_{\mathrm{PAA} 0}$ and $c_{\mathrm{DHA} 0}$, feed rate $F$ chosen to be constant during a run, and feed concentration $c_{\mathrm{D}}^{\text {in }}$. The possible ranges of these independent variables are given in Table 2. Since $c_{\mathrm{D} 0}, c_{\mathrm{P} 0}, F$ and $c_{\mathrm{D}}^{\text {in }}$ have the largest impact on the resulting transient behavior, a $2^{6-2}$ factorial design consisting of 16 experiments is selected. Fewer experimental runs would reduce the validity range and/or the predictive capability of the model, while additional runs would improve them.

\subsection{Various modeling scenarios}

In the following, three different modeling scenarios are presented, each differing in the amount of prior knowledge regarding the reactions. The fluxes, reaction rates and reaction kinetics are identified from noisy concentration measurements.

Scenario 1 In the first scenario, we assume knowledge regarding the existence of reactions (10a)-(10d), including their stoichiometric coefficients. Moreover, it is known that the rates of the reactions $(10 \mathrm{a}),(10 \mathrm{~b})$ and $(10 \mathrm{~d})$ are proportional to the catalyst concentration, see (11).

The reaction fluxes for the various species are obtained from (8) using appropriate regularization. Here, smoothing splines with GCV are used for determining the regularization parameters.

From the time-dependent reaction fluxes $f_{i}^{\mathrm{r}}, i=$ $\{\mathrm{D}, \mathrm{P}, \mathrm{PAA}, \mathrm{DHA}\}$, the time-dependent reaction 
rates $r_{j}, j=\{a, b, c, d\}$, can be calculated using (15a)-(15d). Since the influence of the catalyst on the reaction rates is known, the formal reaction rates $r_{j}^{\star}$ are determined from (11) and (12).

Finally, the concentrations and the reaction rates from one or several runs are correlated as $r_{a}^{\star}=$ $r_{a}^{\star}\left(c_{\mathrm{P}}, c_{\mathrm{D}}\right), r_{b}^{\star}=r_{b}^{\star}\left(c_{\mathrm{D}}\right), r_{c}^{\star}=r_{c}^{\star}\left(c_{\mathrm{D}}\right)$ and $r_{d}^{\star}=$ $r_{d}^{\star}\left(c_{\mathrm{PAA}}, c_{\mathrm{D}}\right)$, as proposed by stoichiometry. A feed-forward neural net with Bayesian regularization as training algorithm and 3 nodes in the hidden layer is utilized.

Scenario 2 In the second scenario, no information regarding the effect of catalyst on the kinetics is postulated. This corresponds to $r_{j}^{\star}=r_{j}$. Otherwise, the procedure is identical to that of Scenario 1.

Scenario 3 We consider the case where little is known a priori about the reaction system. Besides the known desired reaction (10a), there is evidence that diketene (D) and pyrrole $(\mathrm{P})$ are involved in other reactions, including the formation of the dimerization product DHA. Hence, the stoichiometric model

$$
\begin{aligned}
& \mathrm{P}+\mathrm{D} \rightarrow \mathrm{PAA} \\
& \mathrm{D}+\nu_{1} \mathrm{PAA} \rightarrow \nu_{2} \mathrm{DHA}+\mathrm{G}
\end{aligned}
$$

is postulated, where the possible side reactions are lumped into reaction (16b) with the unknown stoichiometric coefficients $\nu_{1}$ and $\nu_{2}$ and some unknown side products $\mathrm{G}$.

From the estimated reaction fluxes, the reaction rates $r_{a}^{\star}(\mathrm{t})$ for Reaction (16a) and $r_{\text {lump }}^{\star}(\mathrm{t})$ for Reaction (16b) as well as the stoichiometric coefficients $\nu_{1}$ and $\nu_{2}$ can be determined as solution of the reconciliation problem:

$$
\begin{aligned}
& f_{\mathrm{D}}^{\mathrm{r}}(t)=\left[-r_{a}^{\star}(t)-r_{\text {lump }}^{\star}(t)\right] V(t) \\
& f_{\mathrm{P}}^{\mathrm{r}}(t)=-r_{a}^{\star}(t) V(t) \\
& f_{\mathrm{PAA}}^{\mathrm{r}}(t)=\left[r_{a}^{\star}(t)-\nu_{1} r_{\text {lump }}^{\star}(t)\right] V(t) \\
& f_{\mathrm{DHA}}^{\mathrm{r}}(t)=\nu_{2} r_{\text {lump }}^{\star}(t) V(t) .
\end{aligned}
$$

The rates $r_{a}^{\star}(\mathrm{t})$ and $r_{\text {lump }}^{\star}(\mathrm{t})$ can subsequently be correlated with the concentrations, as discussed in Scenario 1 .

\subsection{Identification results}

Reaction fluxes and concentrations Exemplarily, the true and estimated reaction fluxes for species $\mathrm{D}$ are shown in Figure 2 (right). Integration of (14a) yields an estimate of the concentration $c_{\mathrm{D}}$, as shown in Figure 2 (left).

Reaction rates For Scenario 1, the estimated rate $r_{b}^{\star}$ in the univariate case is shown in Figure 3, along with training data and true rate.
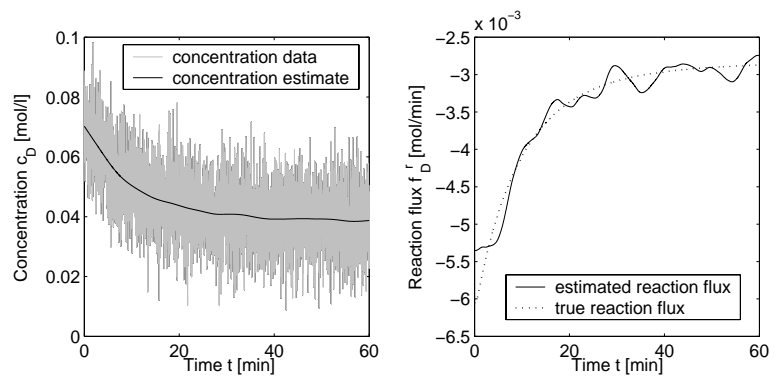

Fig. 2. True and estimated reaction flux $f_{\mathrm{D}}^{\mathrm{r}}$ (right), measured and estimated concentration $c_{\mathrm{D}}$ (left)

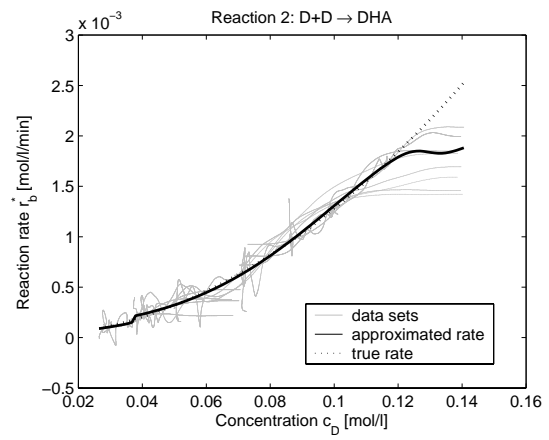

Fig. 3. Estimated reaction rate $r_{b}^{\star}$

Kinetic model The validity range of a model is defined as the smallest $n$-dimensional (e.g. $n=2$ for the bivariate case) box containing all concentration combinations taken for training. The mean and maximum values of the neural net predictions in the validity range are compared to the true (simulated) reaction rates in Table 3.

The predictions obtained in Scenarios 2 and 3 are comparable which can be accredited to the fact that the volume change remains small during the runs (the increase in volume does not exceed $3 \%$ for the low and $9 \%$ for the high feed rate). Presumably, the importance of the catalyst dilution becomes more obvious for large volume changes. Rate $r_{d}^{\star}$, whose value is small compared to the other rates, is mainly influenced by noise and cannot be identified satisfactorily.

For the lumped model in Scenario 3, the main reaction rate $r_{a}^{\star}$ is identified with reasonable accuracy despite the error introduced by lumping all side reactions in (16b). Here, the stoichiometric coefficients $\nu_{1}$ and $\nu_{2}$ were calculated as 0.0028 and 0.2227 , respectively. Since the rates $r_{b}^{\star}, r_{c}^{\star}$ and $r_{d}^{\star}$ were not modeled, they are not identified in this case.

Table 3. Reaction rate prediction errors

\begin{tabular}{llllll}
\hline & & $r_{a}^{\star}$ & $r_{b}^{\star}$ & $r_{c}^{\star}$ & $r_{d}^{\star}$ \\
\hline Scenario 1 & Mean error & 2.95 & 6.15 & 5.16 & 185 \\
& Max. error & 11.24 & 26.42 & 20.92 & 3245 \\
\hline Scenario 2 & Mean error & 4.48 & 7.33 & 4.88 & 117 \\
& Max. error & 15.69 & 25.84 & 18.58 & 466 \\
\hline Scenario 3 & Mean error & 4.64 & - & - & - \\
& Max. error & 62.99 & - & - & - \\
\hline
\end{tabular}


To check the predictive capability of the hybrid model consisting of the mole balance equations and the neural-net-based kinetic laws, concentration trajectories are simulated using (13a)-(13d) and the neural net approximations used to predict them. Ten runs were simulated with experimental conditions chosen randomly within the ranges given in Table 2 . The mean and maximum values of the prediction errors are listed in Table 4.

Table 4. Hybrid model prediction errors

\begin{tabular}{llllll}
\hline & {$[\%]$} & $c_{\mathrm{D}}$ & $c_{\mathrm{P}}$ & $c_{\mathrm{PAA}}$ & $c_{\mathrm{DHA}}$ \\
\hline Scenario 1 & Mean error & 0.81 & 0.11 & 0.40 & 1.10 \\
& Max. error & 2.80 & 0.63 & 1.48 & 5.58 \\
\hline Scenario 2 & Mean error & 0.79 & 0.24 & 0.61 & 1.10 \\
& Max. error & 2.30 & 0.43 & 1.19 & 5.04 \\
\hline Scenario 3 & Mean error & 0.60 & 0.20 & 0.53 & 4.13 \\
& Max. error & 1.91 & 0.42 & 1.28 & 15.58 \\
\hline
\end{tabular}

Hybrid model predictions show excellent agreement with the true (simulated) values. The fact that the model predictions are good even for a poorly estimated reaction rate $r_{d}^{\star}$ illustrates the difficulties experienced in estimating this rate. These results suggest omitting reaction (10d) in the postulated reaction scheme. Comparison of the three scenarios also indicates that the hybrid model derived from the lumped model equations performs nearly as well as the detailed models.

\section{CONCLUSIONS}

This work has proposed an incremental approach for the identification of unknown kinetics in a chemical reactor. The approach consists of: (i) model-free estimation of the reaction flux associated with each species, (ii) calculation of the reaction rates using the (partially) known system stoichiometry, and (iii) identification of kinetic models using neural nets to represent the correlation between reaction rates and concentrations. Here, information on stoichiometry helps choosing the independent variables.

The predictive capability of the hybrid model was very satisfying as were the kinetics identified in the case of known stoichiometry. The errors observed were largely caused by missing initial information regarding the reaction fluxes, a phenomenon that requires further investigation.

It should be emphasized that the proposed incremental modeling approach is by no means limited to the use of neural net submodels. Mechanistic models using target factor analysis, multidimensional sparse grids or multigrid methods may also take advantage of the incremental approach.
This work was partially funded by the Deutsche Forschungsgemeinschaft (DFG) within the Collaborative Research Center (SFB 540) "Modelbased experimental analysis of kinetic phenomena in fluid multiphase reactive systems"

\section{REFERENCES}

Alsmeyer, F., W. Marquardt and G. Olf (2002). A new method for phase equilibrium measurements in reacting mixtures. Fluid Phase Equilibria 203, 31-51.

Bardow, A., W. Marquardt, V. Goeke, H.-J. Koss and K. Lucas (2003). Model-based measurement of diffusion using Raman spectroscopy. AIChE Journal 49(2), 323-334.

Bonvin, D. and D. W. T. Rippin (1990). Target factor analysis for the identification of stoichiometric models. Chemical Engineering Science 45(12), 3417-3426.

Connors, K. A. (1990). Chemical Kinetics: The Study of Reaction Rates in Solution. VCH publishers. New York.

Craven, P. and G. Wahba (1979). Smoothing noisy data with spline functions. Numer. Math. 31, 377-403.

Engl, H. W., M. Hanke and A. Neubauer (1996). Regularization of Inverse Problems. Kluwer Academic Publishers. Dordrecht.

Hansen, P. C. (1998). Rank-deficient and Discrete Ill-posed Problems. SIAM. Philadelphia.

MacKay, D. J. C. (1992). Bayesian interpolation. Neural computation 4(3), 415-447.

Marquardt, W. (2002). Adaptivity in process systems modeling. In: Proc. European Symposium on Computer Aided Process Engineering (ESCAPE-12). Elsevier. The Hague, The Netherlands. pp. $42-56$.

Mhamdi, A. and W. Marquardt (1999). An inversion approach to the estimation of reaction rates in chemical reactors. In: Proc. European Control Conference (ECC'99). Karlsruhe, Germany. Paper F1004-1.

Psichogios, D. C. and L. H. Ungar (1992). A hybrid neural network - first principles approach to process modeling. AIChE Journal 38(10), 1499-1511.

Ruppen, D., D. Bonvin and D. W. T. Rippin (1997). Implementation of adaptive optimal operation for a semi-batch reaction system. Computers Chem. Engng. 22(1-2), 185-199.

Tholudur, A. and W. F. Ramirez (1999). Neuralnetwork modeling and optimization of induced foreign protein production. AIChE Journal 45(8), 1660-1670.

Van Lith, P. F., B. H. L. Betlem and B. Roffel (2002). A structured modeling approach for dynamic hybrid fuzzy first-principles models. J. Proc. Cont. 12, 605-615. 